\title{
COAGULATION PROCESSES WITH GIBBSIAN TIME EVOLUTION
}

\author{
BORIS L. GRANOVSKY ${ }^{* * *}$ AND \\ ALEXANDER V. KRYVOSHAEV, ${ }^{* * *}$ Technion - Israel Institute of Technology
}

\begin{abstract}
We prove that a stochastic process of pure coagulation has at any time $t \geq 0$ a timedependent Gibbs distribution if and only if the rates $\psi(i, j)$ of single coagulations are of the form $\psi(i ; j)=i f(j)+j f(i)$, where $f$ is an arbitrary nonnegative function on the set of positive integers. We also obtain a recurrence relation for weights of these Gibbs distributions that allow us to derive the general form of the solution and the explicit solutions in three particular cases of the function $f$. For the three corresponding models, we study the probability of coagulation into one giant cluster by time $t>0$.
\end{abstract}

Keywords: Stochastic process of coagulation; time dynamics; Gibbs distribution

2010 Mathematics Subject Classification: Primary 82C23

Secondary 60J27; 05A 18

\section{Process of pure coagulation: formulation of the model. Objective and the context of the paper}

We consider a standard model of stochastic coagulation (see, e.g. [7]), viewed as a timecontinuous Markov chain on the finite set $\Omega_{N}$ of partitions $\eta$ of a given integer $N$ :

$$
\Omega_{N}=\left\{\eta=\left(n_{1}, \ldots, n_{N}\right): \sum_{k=1}^{N} k n_{k}=N\right\} .
$$

In the context considered, $N$ is the total number (equivalently, the total mass) of identical particles (molecules, planets, animals, etc.) partitioned into clusters of different sizes, so that $n_{k}$ is the number of clusters of size $k$ in a partition $\eta \in \Omega_{N}$. Infinitesimal in time transitions are coagulations of any two clusters of sizes $i$ and $j$ into one cluster of size $i+j$, resulting in the state transition $\eta \rightarrow \eta^{(i, j)}$. Here $\eta^{(i, j)} \in \Omega_{N}$ codes the state that is obtained from a state $\eta \in \Omega_{N}$, with $n_{i}>0$ and $n_{j}>0$, by a coagulation of any two specific clusters of sizes $i$ and $j$. In the sequel such coagulations are called single and their rates are denoted by $\psi(i, j)$. The following assumptions on the rates $\psi(i, j)$ and on the induced rates $K\left(\eta \rightarrow \eta^{(i, j)}\right)$ of state transitions describe the class of coagulation processes (CPs) under consideration.

- The function $\psi(i, j)$ is nonnegative, symmetric in $i$ and $j$, and is not dependent on $N$.

- The rate $K\left(\eta \rightarrow \eta^{(i, j)}\right)$ is equal to the sum of rates of all single coagulations $\psi(i, j)$ of $n_{i}>0$ groups of size $i$ each with $n_{j}>0$ groups of size $j$ each, so that

$$
K\left(\eta \rightarrow \eta^{(i, j)}\right)=n_{i} n_{j} \psi(i, j), \quad i \neq j, 2 \leq i+j \leq N,
$$

Received 24 August 2010; revision received 18 March 2012.

* Postal address: Department of Mathematics, Technion - Israel Institute of Technology, Haifa 32000, Israel.

** Email address: mar18aa@techunix.technion.ac.il

*** Email address: alexkriv@tx.technion.ac.il 
and

$$
K\left(\eta \rightarrow \eta^{(i, i)}\right)=\frac{n_{i}\left(n_{i}-1\right)}{2} \psi(i, i), \quad 2 \leq 2 i \leq N .
$$

An interpretation of the above expression for the coagulation kernel $K$ in terms of the kinetics of droplets of different masses is given in [22]. CPs with rates of state transitions of the above form are naturally called mean-field models, meaning that at any state $\eta \in \Omega_{N}$, any cluster can coagulate with any other. Hence, given $N$, the distribution of a $\mathrm{CP}=\mathrm{CP}(N)$ at any time $t \geq 0$ is uniquely specified by the initial distribution on the set $\Omega_{N}$ and the rates $\psi(i, j)$.

The history of CPs goes back to 1918 when Smoluchowski formulated a deterministic version of the model of pure coagulation of molecules in chemical kinetics in his seminal paper. Because of the ever growing field of applications and a rich probabilistic context, the study of a variety of versions of the model continues to be a hot topic in the theory of stochastic processes. Marcus [20] was apparently the first to formulate the stochastic version of a CP. A particular case of a pure coagulation, when $\psi(i ; j)=a(i+j)+b, a, b \geq 0$, is known in the literature as the Marcus-Lushnikov stochastic process, while in [1] as well as in some other papers, the name is given to all stochastic CPs with the rates of single coagulations of the form $N^{-1} \psi(i ; j)$ with an arbitrary $\psi(i, j)$. It is important to point out that, in contrast to the latter Marcus-Lushnikov process, the basic assumption of our setting is that the rates of single coagulations do not depend on $N$. The equilibria of some reversible models with rates of coagulation and fragmentation depending on $N$ were studied in [13] and [15].

Let $X_{N}^{(\rho)}(t), t \geq 0$, denote a $\mathrm{CP}(N)$ starting from an initial distribution $\rho$ on $\Omega_{N}$. Our objective is to study the probability distribution (equivalently, the transition probability) $p(\eta, \rho ; t)$ of the process, which is given by

$$
\mathrm{P}\left(X_{N}^{(\rho)}(t)=\eta\right), \quad \eta \in \Omega_{N}, t \geq 0 .
$$

In the sequel we refer in more detail to the literature related to the aforementioned objective.

We now describe the organization of the paper. In Section 2 we formulate our main result which is the characterization of CPs possessing probability distributions (1.1) of Gibbsian type at any time $t \geq 0$. As corollaries of our theorem, we derive the general form of the weights of the aforementioned Gibbs distributions and the explicit expressions for the weights of three particular CP models. We also analyze the behaviour in time of some important functionals of the models. Finally, in Section 3 we explain the name 'Gibbsian' given to the class of distributions considered, describe the linkage to coagulation-fragmentation processes on set partitions, and indicate the relation of Gibbsian distributions to the theory of random combinatorial structures.

\section{Main result}

Recall that $X_{N}^{(\rho)}(t), t \geq 0$, denotes a $\mathrm{CP}(N)$ starting from an initial distribution $\rho$ on $\Omega_{N}$. Our goal is to identify CPs with probability distributions (1.1) of the form

$$
p(\eta, \rho ; t)=C_{N}(t) \prod_{k=1}^{N} \frac{\left(a_{k, N}(t)\right)^{n_{k}}}{n_{k} !}, \quad \eta=\left(n_{1}, \ldots, n_{N}\right) \in \Omega_{N}, t \geq 0 .
$$

Here $C_{N}(t), t \geq 0$, is a time-dependent partition function, and the $a_{k, N}(t) \geq 0, t \geq 0$, are time- and $N$-dependent weights. The distributions on the right-hand side of (2.1) are called 
Gibbsian (equivalently, canonical Gibbsian). We note that, by the above definition, the initial distribution $\rho$ is Gibbsian. By virtue of the mass conservation law, $\sum_{j=1}^{N} j n_{j}=N$, tilting transformations of the weights in (2.1) with an arbitrary function $r_{N}(t)>0, t \geq 0$, i.e.

$$
\tilde{a}_{k, N}(t)=\left(r_{N}(t)\right)^{k} a_{k, N}(t), \quad k=1, \ldots, N, t \geq 0,
$$

and the induced transformation $\tilde{C}_{N}(t)=\left(r_{N}(t)\right)^{-N} C_{N}(t)$ of the partition function result in different representations of the generic Gibbs distribution. In view of this fact we assume in the rest of the paper that the weights $a_{k, N}(t)$ in (2.1) are such that the partition function does not depend on $t \geq 0$, i.e. $C_{N}(t)=C_{N}, t \geq 0$. The latter assumption appears to be of great help for our discussion below.

Our main result is the following characterization.

Theorem 2.1. The probability distributions $p(\eta, \rho ; t)$ of a $C P X_{N}^{(\rho)}(t), t \geq 0$, are of a Gibbsian form (2.1) if and only if the following three conditions are satisfied.

(i) The initial distribution $\rho$ on $\Omega_{N}$ is Gibbsian with arbitrary weights $a_{k, N}=a_{k, N}(0) \geq 0$, $k=1, \ldots, N, N \geq 1$.

(ii) The rates of single coagulations are of the form

$$
\psi(i, j)=i f(j)+j f(i), \quad 1 \leq i+j \leq N,
$$

where $f$ is an arbitrary nonnegative function on the set of positive integers.

(iii) The weights $a_{k, N}(t)$ are defined recursively by

$$
\begin{aligned}
a_{1, N}(t)= & \mathrm{e}^{-(N-1) f(1) t} a_{1, N}, \quad t \geq 0, \\
a_{k, N}(t)= & \int_{0}^{t} \frac{\sum_{i+j=k} a_{i, N}(u) a_{j, N}(u)(i f(j)+j f(i))}{2} \mathrm{e}^{-(N-k) f(k)(t-u)} \mathrm{d} u \\
& +a_{k, N} \mathrm{e}^{-(N-k) f(k) t}, \quad k=2, \ldots, N, t \geq 0,
\end{aligned}
$$

where $a_{k, N} \geq 0$ are constants implied by the initial distribution $\rho$ in $(i)$.

Proof. Our plan is to show that assertions (i)-(iii) are implied by the Kolmogorov forward equations. Suppose that $\eta_{(i, j)}$ is the state that is obtained from a state $\eta \in \Omega_{N}$, with $n_{i+j}>0$, by a fragmentation of some cluster of size $i+j \geq 2$ into two clusters of sizes $i$ and $j$. Then the equations read as

$$
\begin{aligned}
\frac{\mathrm{d}}{\mathrm{d} t} p(\eta, \rho ; t)= & -p(\eta, \rho ; t)\left(\sum_{1 \leq i<j \leq N} n_{i} n_{j} \psi(i, j)+\sum_{i=1}^{N} \frac{n_{i}\left(n_{i}-1\right)}{2} \psi(i, i)\right) \\
& +\sum_{1 \leq i<j \leq N} p\left(\eta_{(i, j)}, \rho ; t\right)\left(n_{i}+1\right)\left(n_{j}+1\right) \psi(i, j) \\
& +\sum_{i=1}^{N} p\left(\eta_{(i, i)}, \rho ; t\right) \frac{\left(n_{i}+1\right)\left(n_{i}+2\right)}{2} \psi(i, i) .
\end{aligned}
$$


First, we assume that (2.1) holds. Substituting $p(\eta, \rho ; t)$ given by (2.1) with weights $a_{k, N}(t)$, such that the partition function does not depend on $t \geq 0$, we obtain

$$
\begin{aligned}
\frac{\mathrm{d}}{\mathrm{d} t} p(\eta, \rho ; t)= & -p(\eta, \rho ; t)\left(\sum_{1 \leq i<j \leq N} n_{i} n_{j} \psi(i, j)+\sum_{i=1}^{N} \frac{n_{i}\left(n_{i}-1\right)}{2} \psi(i, i)\right) \\
& +p(\eta, \rho ; t)\left(\sum_{1 \leq i<j \leq N} \frac{a_{i, N}(t) a_{j, N}(t)}{a_{i+j, N}(t)} \frac{n_{i+j}}{\left(n_{i}+1\right)\left(n_{j}+1\right)}\right. \\
& \left.+\sum_{i=1}^{N} \frac{a_{i, N}^{2}(t)}{a_{2 i, N}(t)} \frac{n_{2 i}}{\left(n_{i}+1\right)\left(n_{i}+2\right)} \frac{\left(n_{i}+1\right)\left(n_{i}+2\right)}{2} \psi(i, i)\right),
\end{aligned}
$$

which can be written as

$$
\begin{aligned}
\frac{\mathrm{d} p(\eta, \rho ; t) / \mathrm{d} t}{p(\eta, \rho ; t)}= & \sum_{1 \leq i, j \leq N} \frac{a_{i, N}(t) a_{j, N}(t)}{2 a_{i+j, N}(t)} n_{i+j} \psi(i, j) \\
& -\left(\sum_{1 \leq i<j \leq N} n_{i} n_{j} \psi(i, j)+\sum_{i=1}^{N} \frac{n_{i}\left(n_{i}-1\right)}{2} \psi(i, i)\right), \quad \eta \in \Omega_{N}, t \geq 0 .
\end{aligned}
$$

In view of (2.1) and the time independence of the partition function, we proceed as

$$
\begin{aligned}
\sum_{k=1}^{N} \frac{a_{i, N}^{\prime}(t)}{a_{i, N}(t)} n_{i}= & \sum_{1 \leq i, j \leq N} \frac{a_{i, N}(t) a_{j, N}(t)}{2 a_{i+j, N}(t)} n_{i+j} \psi(i, j) \\
& -\left(\sum_{1 \leq i<j \leq N} n_{i} n_{j} \psi(i, j)+\sum_{i=1}^{N} \frac{n_{i}\left(n_{i}-1\right)}{2} \psi(i, i)\right), \quad \eta \in \Omega_{N}, t \geq 0 .
\end{aligned}
$$

Finally, we have

$$
\begin{aligned}
\sum_{k=1}^{N} \frac{a_{i, N}^{\prime}(t)}{a_{i, N}(t)} n_{i}= & \left(\sum_{k=2}^{N} \frac{\sum_{i+j=k} a_{i, N}(t) a_{j, N}(t) \psi(i, j)}{2 a_{k, N}(t)} n_{k}\right) \\
& -\frac{1}{2}\left(\sum_{1 \leq i, j \leq N} n_{i} n_{j} \psi(i, j)-\sum_{i=1}^{N} \psi(i, i) n_{i}\right), \quad \eta \in \Omega_{N}, t \geq 0 .
\end{aligned}
$$

We now rewrite the last equation as

$$
\frac{1}{2}\left(\sum_{1 \leq i, j \leq N} n_{i} n_{j} \psi(i, j)\right)=\sum_{k=1}^{N} A_{k, N}(t) n_{k}, \quad t \geq 0, \text { for all } \eta=\left(n_{1}, \ldots, n_{N}\right) \in \Omega_{N},
$$

where

$$
A_{k, N}(t)=\frac{\sum_{i+j=k} a_{i, N}(t) a_{j, N}(t) \psi(i, j)}{2 a_{k, N}(t)}-\frac{a_{k, N}^{\prime}(t)}{a_{k, N}(t)}+\frac{1}{2} \psi(k, k), \quad k=1, \ldots, N,
$$


assuming that, for $k=1$, the sum on the right-hand side of the last expression equals 0 . Applying the mass conservation law, (2.5) becomes

$$
\frac{1}{2}\left(\sum_{1 \leq i, j \leq N} n_{i} n_{j} \psi(i, j)\right)=\frac{1}{2 N} \sum_{1 \leq i, j \leq N} n_{i} n_{j}\left(j A_{i, N}(t)+i A_{j, N}(t)\right)
$$

for all $\eta=\left(n_{1}, \ldots, n_{N}\right) \in \Omega_{N}$ and $t \geq 0$, from which, using the assumed symmetry of the function $\psi(i, j)$, we derive the unique form of $\psi$ :

$$
\psi(i, j)=\frac{1}{N}\left(j A_{i, N}(t)+i A_{j, N}(t)\right), \quad\left(n_{1}, \ldots, n_{N}\right) \in \Omega_{N}, t \geq 0 .
$$

Consequently,

$$
\psi(i, i)=\frac{2}{N} i A_{i, N}(t), \quad t \geq 0, i \geq 1,
$$

which imposes the following necessary and sufficient conditions on the coefficients $A_{i, N}(t)$ :

$$
N^{-1} A_{i, N}(t)=: f(i), \quad i \geq 1,
$$

does not depend on $N \geq 1$ and $t \geq 0$. By virtue of this condition and (2.7), we find that

$$
\psi(i, i)=2 i f(i), \quad \psi(i, j)=i f(j)+j f(i),
$$

which proves the necessity of part (ii) of the theorem. It remains to demonstrate that the weights $a_{i, N}(t)$ can be found recursively from (2.6) and (2.8). First, we recover $a_{1, N}(t)$ :

$$
\begin{gathered}
N^{-1}\left(-\frac{a_{1, N}^{\prime}(t)}{a_{1, N}(t)}+f(1)\right)=f(1), \quad t \geq 0, \\
a_{1, N}(t)=a_{1, N} \mathrm{e}^{-(N-1) f(1) t}, \quad t \geq 0 .
\end{gathered}
$$

Here $a_{1, N}(0)=a_{1, N}>0$ is a constant, given by the initial distribution $\rho$. For $k \geq 2,(2.8)$ takes the form

$$
N^{-1}\left(\frac{\sum_{i+j=k} a_{i, N}(t) a_{j, N}(t) \psi(i, j)}{2 a_{k, N}(t)}-\frac{a_{k, N}^{\prime}(t)}{a_{k, N}(t)}+k f(k)\right)=f(k) .
$$

Observing that the convolution term

$$
M_{k, N}(t):=\sum_{i+j=k} a_{i, N}(t) a_{j, N}(t) \psi(i, j)=\sum_{i+j=k} a_{i, N}(t) a_{j, N}(t)(i f(j)+j f(i)), \quad t \geq 0,
$$

does not depend on $a_{k, N}(t)$, we arrive at the following first-order differential equation for $a_{k, N}(t)$ :

$$
a_{k, N}^{\prime}(t)=\frac{M_{k, N}(t)}{2}-(N-k) f(k) a_{k, N}(t), \quad k=2, \ldots, N .
$$

Solving it we obtain the recurrence relation that conforms to part (iii) of the theorem:

$$
a_{k, N}(t)=\mathrm{e}^{-(N-k) f(k) t}\left(\int_{0}^{t} \frac{M_{k, N}(u)}{2} \mathrm{e}^{(N-k) f(k) u} \mathrm{~d} u+a_{k, N}\right), \quad k=2, \ldots, N, t \geq 0 .
$$

Now the validation that, under conditions (i)-(iii), (2.1) is the solution of the Kolmogorov equations (2.4) is obvious.

Notes. (i) An obvious consequence of Theorem 2.1 is that a CP with given rates $\psi(i, j)$ of the form (2.2), starting from a non-Gibbsian initial distribution, does not possess Gibbsian 
transition probabilities. In particular, if the aforementioned process starts from a mixture of Gibbs distributions then its transition probabilities (1.1) are the corresponding mixtures of Gibbs distributions.

(ii) The differential recurrence (2.3) was originally derived by Lushnikov [18], [19] where the weights $a_{k, N}(t), t \geq 0$, were associated with the generating function for the probabilities $p(\eta, \rho ; t), t \geq 0$. Buffet and Pulé [5] proved the core fact that, given $\psi(i, j)=i f(j)+j f(i)$, the Kolmogorov equations are solved by Gibbsian distributions $p(\eta, \rho ; t)$ with weights satisfying the differential recurrence (2.3). Our result strengthens that of [5] in the following four directions.

- We establish the necessity of the form (2.2) of rates for Gibbsian transition probabilities (1.1).

- The study in [5] is limited to Gibbs distributions with partition functions $C_{N}=1, N \geq 1$, which substantially restrict the class of possible initial distributions. For example, under the above restriction, the initial Gibbs distribution with constant weights $a_{k, N}=1$ is not allowed, since in this case the explicit expression for $C_{N}$ as a function of $N$ is not known. So, the trick with the tilting transformation cannot be applied.

- Our theorem is proven for the case of a nonnegative (rather than positive) function $f$, which enables us to treat CPs like those of Becker-Döring, which are defined in Corollary 2.4 below.

- Solving in Corollary 2.1 below the recurrence relation (2.3), we find a general form of the weights $a_{k, N}(t)$.

(iii) In [5, p. 1047] it was noted that if $N$ is a multiple of an integer $q \geq 1$ then the initial distribution $\rho$ concentrated on the partition of $N$ into $N / q$ groups, each of size $q$, is Gibbsian, with

$$
a_{q, N}>0, \quad a_{k, N}=0, \quad N \geq k \neq q .
$$

An important particular case of the above measures is given by $q=1$, which in chemistry corresponds to what is called total dissipation. In this case the initial distribution $\rho$ is concentrated on the partition $(N, 0, \ldots, 0)$ and $C_{N}=N ! / a_{1, N}^{N}$, where $a_{1, N}>0$ is arbitrary, while $a_{k, N}=0, k=2, \ldots, N$. A closer look at the above example in [5] shows that a class of Gibbs initial distributions encompasses a variety of (but not all) measures concentrated on single partitions of $N$. We give an example of one such measure. Let $N=l+m$, where $0<l<m$ and $N$ is not divisible by $l$. This ensures that $\eta^{*}=\left(n_{1}^{*}, \ldots, n_{N}^{*}\right)$, where $n_{l}^{*}=n_{m}^{*}=1$, is the unique partition of $N$ with $n_{l}>0$ and $n_{m}>0$. Hence, the measure $\rho$ such that $\rho\left(\eta^{*}\right)=1$ can be viewed as a Gibbs distribution with arbitrary weights $a_{l, N}>0$ and $a_{m, N}>0$, all other weights equal to 0 , and partition function $C_{N}=\left(a_{l, N} a_{m, N}\right)^{-1}$.

In the rest of the paper, we assume that the initial distribution is the total dissipation with $a_{1, N}=a_{1, N}(0)=1, N \geq 1$, so that $C_{N}=N !, N \geq 1$.

Recurrence relation (2.3) allows us to find a general form of the parameters $a_{k, N}(t)$ for an arbitrary function $f \geq 0$. To this end, we need more notation. Let

$$
\eta^{(j)}=\left\{\left(l_{1}^{(j)}, \ldots, l_{j}^{(j)}\right): \sum_{s=1}^{j} s l_{s}^{(j)}=j\right\}
$$


be a partition from the set $\Omega_{j}$ of integer partitions of $j$, and set

$$
q\left(\eta^{(j)} ; N\right):=\sum_{s=1}^{j} l_{s}^{(j)}(N-s) f(s)
$$

for $j=1, \ldots, N$ and $f \geq 0$.

Corollary 2.1. (The general form of the weights $a_{k, N}(t)$.) For given $N$ and a function $f \geq 0$, the solution $a_{k, N}(t)$ of (2.3) has the form

$$
a_{k, N}(t)=\sum_{\eta^{(k)} \in \Omega_{k}} B\left(\eta^{(k)} ; N\right) \exp \left(-q\left(\eta^{(k)} ; N\right) t\right), \quad t \geq 0, k=1, \ldots, N
$$

where the coefficients $B\left(\eta^{(k)} ; N\right)$ do not depend on $t \geq 0$.

Proof. We proceed by induction on $1 \leq k \leq N$. Recalling our assumption that

$$
a_{k ; N}:=a_{k ; N}(0)= \begin{cases}1 & \text { if } k=1 \\ 0 & \text { if } k=2, \ldots, N\end{cases}
$$

we obtain, from (2.3),

$$
a_{1, N}(t)=\mathrm{e}^{-(N-1) f(1) t}, \quad t \geq 0,
$$

which is of the form $(2.10)$ with $q\left(\eta^{(1)} ; N\right)=(N-1) f(1)$ and $B\left(\eta^{(1)} ; N\right)=1$ for $N \geq 1$. Consequently, we derive, from (2.3),

$$
a_{2 ; N}(t)=\frac{f(1)}{2(N-1) f(1)-(N-2) f(2)}\left(\mathrm{e}^{-f(2)(N-2) t}-\mathrm{e}^{-2 f(1)(N-1) t}\right), \quad t \geq 0,
$$

which is of the form (2.10) induced by the two partitions $(2,0)$ and $(0,1)$ of 2 . Now assume that (2.10) holds for $a_{j, N}, j=1, \ldots, k-1$. Substituting into (2.3) the expression for $a_{j, N}(u), j=$ $1, \ldots, k-1$ given in (2.10), the claim for $a_{k, N}(t)$ follows from the fact that, for any given $\eta^{(j)} \in \Omega_{j}$ and $\eta^{(k-j)} \in \Omega_{k-j}$,

$$
q\left(\eta^{(j)} ; N\right)+q\left(\eta^{(k-j)} ; N\right)=q\left(\eta^{(k)} ; N\right),
$$

where $\eta^{(k)} \in \Omega_{k}$ is the partition of $k$ obtained by merging the two partitions $\eta^{(j)}$ and $\eta^{(k-j)}$.

Corollary 2.2. (Additive rates of coagulation: $\psi(i, j)=(i+j) v, v>0$.) In this case the probability distributions $p(\eta, \rho ; t)$ can be found explicitly. Namely,

$$
\begin{aligned}
p(\eta, \rho ; t)= & N ! \mathrm{e}^{-N(r-1) v t}\left(1-\mathrm{e}^{-N v t}\right)^{N-r} N^{-(N-r)} \\
& \times \prod_{k=1}^{N} \frac{k^{(k-1) n_{k}}}{(k !)^{n_{k}} n_{k} !}, \quad t \geq 0,\left(n_{1}, \ldots, n_{N}\right) \in \Omega_{r, N},
\end{aligned}
$$

where $\Omega_{r, N}=\left\{\eta=\left(n_{1}, \ldots, n_{N}\right) \in \Omega_{N}: n_{1}+\cdots+n_{N}=r, 1 \leq r \leq N\right\}$ denotes the set of all partitions of $N$ with $r$ summands (equivalently, clusters).

Proof. This case conforms to (2.2) with $f(j)=v>0, j \geq 1$. We will seek the solution of recurrence relation (2.3) in the form

$$
a_{k, N}(t)=\mathrm{e}^{-(N-k) v t}\left(1-\mathrm{e}^{-N v t}\right)^{k-1} v_{k, N}, \quad k=1, \ldots, N, N \geq 1,
$$


with constants $v_{k, N}>0$ that will be determined from (2.3). For $k=1$, (2.12) reduces to (2.9) with $v_{1, N}=a_{1, N}=1$. Next, by the induction argument on $k \geq 1$, (2.3) gives

$$
\begin{aligned}
a_{k, N}(t) & =\mathrm{e}^{-(N-k) v t}\left(\int_{0}^{t} \frac{v k \mathrm{e}^{-(2 N-k) v u}\left(1-\mathrm{e}^{-N v u}\right)^{k-2} \mathrm{e}^{(N-k) v u}}{2} \mathrm{~d} u\right) \sum_{i+j=k} v_{i, N} v_{j, N} \\
& =\mathrm{e}^{-(N-k) v t}\left(1-\mathrm{e}^{-N v t}\right)^{k-1} \frac{N^{-1} k}{2(k-1)} \sum_{i+j=k} v_{i, N} v_{j, N} .
\end{aligned}
$$

In view of (2.12), this leads to the following recurrence relation for $v_{k, N}$ :

$$
v_{k, N}=\frac{N^{-1} k}{2(k-1)} \sum_{i+j=k} v_{i, N} v_{j, N}, \quad k=2, \ldots, N-1 .
$$

This is a particular case of Recursion (2.33) of [12], whose solution is

$$
v_{k, N}=N^{-(k-1)} \frac{k^{k-1}}{k !}, \quad k=1, \ldots, N
$$

(see Equation (2.39) of [12]).

From (2.11) we find the probability of coagulation, $p_{\mathrm{coag}, N}(t)$, into one giant cluster of size $N$ by time $t>0$ :

$$
p_{\text {coag }, N}(t)=\left(1-\mathrm{e}^{-N v t}\right)^{N-1} \rightarrow 1 \quad \text { as } N \rightarrow \infty \text { for } t>0 .
$$

This says that a strong gelation phenomenon holds at any time $t>0$ as $N \rightarrow \infty$.

We discuss below the probabilistic meaning of distribution (2.11). We rewrite (2.11) as

$$
\begin{aligned}
p(\eta, \rho ; t)= & \left(\begin{array}{c}
N-1 \\
r-1
\end{array}\right) \mathrm{e}^{-N(r-1) v t}\left(1-\mathrm{e}^{-N v t}\right)^{N-r} B_{r, N}^{-1} \\
& \times \prod_{k=1}^{N} \frac{k^{(k-1) n_{k}}}{(k !)^{n_{k}} n_{k} !}, \quad t \geq 0,\left(n_{1}, \ldots, n_{n}\right) \in \Omega_{r, N},
\end{aligned}
$$

where $B_{r, N}^{-1}=N(r-1) !(N-r) ! N^{-(N-r)}$. It was noted in [12] that the sequence of weights $k^{k-1} / k !, k \geq 1$, is a particular case of weight sequences satisfying the Gnedin-Pitman condition of exchangeability of Gibbs set partitions [9]. It is easy to see that

$$
\left(\begin{array}{c}
N-1 \\
r-1
\end{array}\right) \mathrm{e}^{-N(r-1) v t}\left(1-\mathrm{e}^{-N v t}\right)^{N-r}
$$

in (2.13) expresses the transition probabilities of a pure death process with rates $\mu_{r, N}=(r-1) \times$ $v N, r=1, \ldots, N$, as in Equation (2.17) of [12]. Note that the aforementioned transition probabilities are binomial distributions with time-dependent probabilities of success, while $B_{r, N}$ in (2.13) is the $(N, r)$ partial Bell polynomial (see Equation (2.37) of [12] for more details). In the context of our model $\left(B_{r, N}\right)^{-1}$ serves as the partition function for the conditional distribution $\mathrm{P}\left(X_{N}^{(\rho)}(t)=\eta|| X_{N}^{(\rho)}(t) \mid=r\right)$, which is the microcanonical Gibbs distribution on $\Omega_{r, N}$, with weights $k^{k-1} / k$ ! that are independent of $N$ and $t$. In view of this, we conclude from (2.13) that the distribution of the number of clusters $\left|X_{N}^{(\rho)}(t)\right|:=n_{1}(t)+\cdots+n_{N}(t)$ at time $t$ is binomial:

$$
\mathrm{P}\left(\left|X_{N}^{(\rho)}(t)\right|=r\right)=\left(\begin{array}{c}
N-1 \\
r-1
\end{array}\right) \mathrm{e}^{-N(r-1) v t}\left(1-\mathrm{e}^{-N v t}\right)^{N-r}, \quad t \geq 0 .
$$


This fact was originally proven by Lushnikov (see Equation (49) of [18]). It is interesting that in the case of the Marcus-Lushnikov CP with the $N$-dependent additive kernel, the distribution of $\left|X_{N}^{(\rho)}(t)\right|$ is also binomial, but with a different parameter. The latter distribution was derived by Aldous [1], from the interpretation of the process as the vector of the sizes of the continium random tree.

Corollary 2.3. (Multiplicative rates of coagulation: $\psi(i, j)=2 i j$.) In the case considered, $f(i)=i, i \geq 1$, so that, under the assumed initial distribution, (2.3) becomes

$a_{1, N}(t)=\mathrm{e}^{-(N-1) t}, \quad t \geq 0, N \geq 1$,
$a_{k, N}(t)=\mathrm{e}^{-(N-k) k t} \int_{0}^{t} \mathrm{e}^{k^{2} u} \sum_{i+j=k}\left(i a_{i, N}(u) \mathrm{e}^{N i u}\right)\left(j a_{j, N}(u) \mathrm{e}^{N j u}\right) \mathrm{d} u, \quad k=2, \ldots, N, t \geq 0$.

Defining

$$
i a_{i, N}(u) \mathrm{e}^{N i u}=b_{i, N}(u),
$$

we arrive at the following recurrence relation:

$$
\begin{gathered}
b_{1, N}(t)=\mathrm{e}^{t}, \quad t \geq 0, N \geq 1, \\
b_{k, N}(t)=k \mathrm{e}^{k^{2} t} \int_{0}^{t} \mathrm{e}^{-k^{2} u} \sum_{i+j=k} b_{i, N}(u) b_{j, N}(u) \mathrm{d} u, \quad k=2, \ldots, N, t \geq 0 .
\end{gathered}
$$

The important fact is that the function $b_{1, N}(t)$ and, consequently, by virtue of (2.14), the functions $b_{k, N}(t)$ do not depend on $N: b_{k, N}(t)=b_{k}(t), t \geq 0$. Thus, in the case considered,

$$
p(\eta, \rho ; t)=N ! \mathrm{e}^{-N^{2} t} \prod_{k=1}^{N} \frac{\left(b_{k}(t)\right)^{n_{k}}}{k^{n_{k}} n_{k} !}, \quad \eta=\left(n_{1}, \ldots, n_{N}\right) \in \Omega_{N}, t \geq 0,
$$

where the $b_{k, N}(t)$ are defined in (2.14). The right-hand side of (2.15) can be viewed as the Gibbs distribution on $\Omega_{N}$ with the partition function $N ! \mathrm{e}^{-N^{2} t}$ and the weights $b_{k}(t) / k, k \geq 1$ and $t \geq 0$, not depending on $N$. This allows us to employ the known exponential relation (see, e.g. [7] for references) between the generating functions $H(t ; x)$ and $V(t ; x)$ for the sequences $h_{k}(t):=\mathrm{e}^{k^{2} t} / k !, k \geq 0$ and $t \geq 0$, and $v_{k}(t):=b_{k}(t) / k, k \geq 1$ and $t \geq 0$, respectively:

$$
H(t ; x)=\mathrm{e}^{V(t ; x)}, \quad V(t ; x)=\sum_{k \geq 1} v_{k}(t) x^{k}, \quad H(t ; x)=\sum_{k \geq 0} \frac{\mathrm{e}^{k^{2} t}}{k !} x^{k}, \quad \text { for } t \geq 0 .
$$

We note that the radius of convergence of the power series $H(t ; x)$ is 0 and that, by virtue of the exponential relation (2.16), the same is true for the series $V(t ; x)$. This says that the two power series should be treated as formal power series. From the exponential relation (2.16), it is easy to derive the following recurrence relation between the sequences $\left\{v_{k}(t)\right\}$ and $\left\{h_{k}(t)\right\}$ :

$$
h_{0}(t) \equiv 1, \quad(n+1) h_{n+1}(t)=\sum_{k=0}^{n}(k+1) v_{k+1}(t) h_{n-k}(t), \quad n=0,1, \ldots, t \geq 0 .
$$

It goes without saying that (2.14) and (2.17) are equivalent. However, (2.17) is much more convenient for the study of the asymptotics of $v_{k}(t)$ as $k \rightarrow \infty$. Based on the fact that the 
functions $h_{k}(t)=\mathrm{e}^{k^{2} t} / k$ ! grow very rapidly with $k$ for any fixed $t>0$, we will demonstrate that the solution of (2.17) is given by

$$
v_{k}(t) \sim h_{k}(t) \quad \text { as } k \rightarrow \infty \text { for } t>0 .
$$

First, we see from (2.17) that

$$
v_{n+1}(t) \leq h_{n+1}(t), \quad t \geq 0, n=0,1, \ldots
$$

This implies that

$$
(n+1) v_{n+1} \geq(n+1) h_{n+1}(t)-\sum_{k=0}^{n-1}(k+1) h_{k+1}(t) h_{n-k}(t), \quad t \geq 0,
$$

and, consequently,

$$
\frac{v_{n+1}(t)}{h_{n+1}(t)} \geq 1-\sum_{k=0}^{n-1} \frac{(k+1) h_{k+1}(t) h_{n-k}(t)}{(n+1) h_{n+1}(t)}, \quad t \geq 0 .
$$

This, together with (2.19), says that, for the proof of (2.18), we should validate the limit

$$
\sum_{k=0}^{n-1} \frac{(k+1) h_{k+1}(t) h_{n-k}(t)}{(n+1) h_{n+1}(t)}=\mathrm{e}^{-2 n t} \sum_{k=0}^{n-1}\left(\begin{array}{l}
n \\
k
\end{array}\right) \mathrm{e}^{-2 k(n-k-1) t} \rightarrow 0 \quad \text { as } n \rightarrow \infty
$$

for any $t>0$. We have

$$
\begin{aligned}
\mathrm{e}^{-2 n t} \sum_{k=0}^{[n / 2]}\left(\begin{array}{l}
n \\
k
\end{array}\right) \mathrm{e}^{-2 k(n-k-1) t} & =\mathrm{e}^{-2 n t}\left(1+\sum_{k=1}^{[n / 2]} \frac{1}{k !} \prod_{j=0}^{k-1}(n-j) \mathrm{e}^{-2(n-k-1) t}\right) \\
& \leq \mathrm{e}^{-2 n t}\left(1+\sum_{k=1}^{[n / 2]} \frac{1}{k !} n \mathrm{e}^{-2(n-k-1) t}\right) \\
& \rightarrow 0 \quad \text { as } n \rightarrow \infty
\end{aligned}
$$

In view of the relation $\left(\begin{array}{l}n \\ k\end{array}\right)=\left(\begin{array}{c}n \\ n-k\end{array}\right)$, the same limit can be proven for the sum

$$
\mathrm{e}^{-2 n t} \sum_{k=[n / 2]+1}^{n-1}\left(\begin{array}{l}
n \\
k
\end{array}\right) \mathrm{e}^{-2 k(n-k-1) t}, \quad t>0 .
$$

This completes the proof of (2.18). Equipped with this result we are now in a position to study the time dynamics of the clustering of groups of different sizes as $N \rightarrow \infty$ and $t>0$ is fixed. With an obvious abuse of notation, let the random variable $n_{k, N}(t)$ be the number of groups of size $k$ at time $t>0$. By the known formulae for the functionals of the Gibbs distribution considered (see, e.g. [7]), we obtain, for any fixed moment of time $t>0$,

$$
\begin{gathered}
\mathrm{E} n_{k, N}(t)=v_{k}(t) \frac{h_{N-k}(t)}{h_{N}(t)}, \quad k=1, \ldots, N . \\
\operatorname{var} n_{k, N}(t)=v_{k}^{2}(t)\left(\frac{h_{N-2 k}(t)}{h_{N}(t)}-\left(\frac{h_{N-k}(t)}{h_{N}(t)}\right)^{2}\right)+v_{k}(t) \frac{h_{N-k}(t)}{h_{N}(t)},
\end{gathered}
$$


and

$$
\begin{aligned}
& \operatorname{cov}\left(n_{k, N}(t), n_{l, N}(t)\right) \\
& \quad=v_{k}(t) v_{l}(t)\left(\frac{h_{N-k-l}(t)}{h_{N}(t)}-\frac{h_{N-k}(t) h_{N-l}(t)}{h_{N}^{2}(t)}\right), \quad k \neq l=1,2 \ldots, N .
\end{aligned}
$$

Substituting $h_{k}(t)=\mathrm{e}^{k^{2} t} / k$ !, it is easy to show that the three quantities tend to 0 as $N \rightarrow \infty$ for any fixed $k, l$, and $t>0$. On the other hand, applying (2.18), we conclude that, at any time $t>0$,

$$
\mathrm{E} n_{N, N}(t) \rightarrow 1 \quad \text { as } N \rightarrow \infty \text {. }
$$

This means that $p_{\text {coag, } N}(t) \rightarrow 1$ as $N \rightarrow \infty$ at any moment $t>0$, which is equivalent to saying that a strong form of gelation occurs during all time evolutions of the process.

Unlike in our case, the Marcus-Lushnikov process with the $N$-dependent multiplicative kernel exhibits gelation only after time $t=1$. This fact was proven in [5]. Also, note that in [6] the aforementioned Marcus-Lushnikov process was represented as a random graph process, which among other things allowed a version of (2.15) to be obtained for the case considered. The survey by Aldous [1] enlightens this interesting connection to random graphs. Finally, we note that a properly time-space rescaled Marcus-Lushnikov CP with multiplicative kernel converges to a limit process called the standard multiplicative coalescence (see [1]). This fact facilitates the study of the emergence of the giant cluster. Regarding (2.20), it is in order to observe that the formulae are not valid in the general case of $N$-dependent weights $a_{k, N}(t)$. This can be seen from the derivation of (2.20) (see, e.g. [7]).

Corollary 2.4. (Becker-Döring pure coagulation process.) This CP is a stochastic version of the Becker-Döring kinetic equations proposed in 1935 to model a variety of phenomena in which only coagulations with monomers (equivalently, clusters of size 1) are allowed; see, e.g. [3] for references. Formally,

$$
\psi(i, j)=0 \quad \text { if } \min \{i, j\}>1 .
$$

Clearly, the rates of the process are of the form (2.2) if and only if the function $f$ has the form

$$
f(i)= \begin{cases}0 & \text { if } i>1 \\ v>0 & \text { if } i=1\end{cases}
$$

which leads to the rates

$$
\psi(i, 1)=\psi(1, i)= \begin{cases}i v & \text { if } i>1 \\ 2 v & \text { if } i=1\end{cases}
$$

Correspondingly, (2.3) takes the form

$$
\begin{gathered}
a_{1, N}(t)=\mathrm{e}^{-(N-1) v t}, \quad t \geq 0, \\
a_{k, N}(t)=(k-1) v \int_{0}^{t} \mathrm{e}^{-(N-1) v u} a_{k-1, N}(u) \mathrm{d} u, \quad k=2, \ldots, N, t \geq 0 .
\end{gathered}
$$

From (2.10), it is not difficult to show that in the case considered $a_{k, N}(t)$ is a polynomial of degree $k$ in $z(t)=\mathrm{e}^{-(N-1) v t}, t \geq 0$ :

$$
a_{k, N}(t)=\sum_{i=0}^{k} m_{i, k} \mathrm{e}^{-i(N-1) v t}, \quad k=1, \ldots, N, t \geq 0 .
$$


By (2.4), the coefficients $m_{i, k}=m_{i, k, N}$ are defined recursively by

$$
m_{i, k}=-\frac{(k-1) m_{i-1, k-1}}{i(N-1)}, \quad i=1, \ldots, k, k \geq 2, \quad m_{0, k}=-\sum_{i=1}^{k} m_{i, k}, \quad k \geq 2,
$$

with the initial conditions

$$
m_{0,1}=0, \quad m_{1,1}=1, \quad m_{0,2}=a_{2, N}(\infty)=\frac{1}{2(N-1)}, \quad m_{2,2}=-\frac{1}{2(N-1)} .
$$

The recurrence relation in (2.22) is validated by the induction argument

$$
\begin{gathered}
(k-1) v \int_{0}^{t} \mathrm{e}^{-(N-1) v u}\left(\sum_{i=0}^{k-1} m_{i, k-1} \mathrm{e}^{-i(N-1) v u}\right) \mathrm{d} u \\
=(k-1) v \int_{0}^{t}\left(\sum_{i=1}^{k} m_{i-1, k-1} \mathrm{e}^{-i(N-1) v u}\right) \mathrm{d} u \\
=(k-1) v \sum_{i=1}^{k} \frac{m_{i-1, k-1}}{i(N-1) v}\left(1-\mathrm{e}^{-i(N-1) v t}\right) \\
=a_{k, N}(t), \quad k=2, \ldots, N, t \geq 0 .
\end{gathered}
$$

Solving (2.22), we find the explicit expressions for the coefficients $m_{i, k}$ :

$$
\begin{gathered}
m_{k-1, k}=0, \quad k \geq 1, \quad m_{k, k}=\frac{(-1)^{k-1}}{k(N-1)^{k-1}}, \quad k \geq 1, \\
m_{i, k}=\frac{(-1)^{i}(k-1) !}{i !(N-1)^{k-1}(k-i)(k-i-2) !}, \quad k-i \geq 2 .
\end{gathered}
$$

Consequently, we see that the probability of appearance of one giant cluster of size $N$ appearing decays 'almost' exponentially to 0 as $N \rightarrow \infty$ at any time $t>0$, i.e.

$$
p_{\text {coag }, N}(t)=N ! a_{N, N}(t) \sim N ! m_{0, N}=\frac{(N-1) !}{(N-1)^{N-2}} \sim \sqrt{2 \pi}(N-1)^{3 / 2} \mathrm{e}^{-(N-1)},
$$

where the first ' $\sim$ ' is due to the last relation in (2.22).

In contrast to the models in Corollaries 2.2 and 2.3, the Becker-Döring CP has a nontrivial equilibrium distribution (equivalently, measure) $\mu_{N}(\eta)$, which is given by the weights $a_{k, N}(\infty)$. We have

$$
a_{1, N}(\infty)=0, \quad a_{k, N}(\infty)=m_{0, k}=\frac{k-1}{k(N-1)^{k-1}}, \quad k \geq 2,
$$

so that

$$
\mu_{N}(\eta)=N !(N-1)^{-(N-|\eta|)} \prod_{k=1}^{N}\left(\frac{k-1}{k}\right)^{n_{k}} \frac{1}{n_{k} !}, \quad \eta \in \Omega_{N},
$$

with the convention that $0^{0}=1$, where $|\eta|=n_{1}+\cdots+n_{N}$ is the number of clusters in $\eta$. It follows from (2.24) that $\mu_{N}(\eta)=0$ for all $\eta=\left(n_{1}, \ldots, n_{N}\right) \in \Omega_{N}$ with $n_{1}>0$. 
Finally, we derive from (2.24) the conditional distribution, given $|\eta|$, at the equilibrium of the process (equivalently, the microcanonical distribution at equilibrium):

$$
\mu_{N}(\eta|| \eta \mid=l)=\left(B_{l, N}\right)^{-1} \prod_{k=1}^{N}\left(\frac{k-1}{k}\right)^{n_{k}} \frac{1}{n_{k} !}, \quad \eta=\left\{\left(n_{1}, \ldots, n_{N}\right) \in \Omega_{N}:|\eta|=l \leq N\right\},
$$

where $B_{l, N}=\mathrm{P}(|\eta|=l)$ is the $(l, N)$ partial Bell polynomial on the set of all partitions of $N$ with $n_{1}=0$, induced by the weights $v_{k}:=(k-1) / k$ not depending on $N$.

\section{CPs with canonical and microcanonical Gibbs distributions}

Distribution (2.1) can be written as

$$
p(\eta, \rho ; t)=C_{N}(t) \exp \left(-\sum_{i=1}^{N}\left(-n_{i} \log a_{i, N}(t)+\log \left(n_{i} !\right)\right)\right), \quad \eta \in \Omega_{N}, t \geq 0 .
$$

This shows that in the context of statistical physics, (2.1) conforms to the canonical Gibbs distribution with time-dependent potentials $H_{k}$ of the following special form:

$$
\begin{gathered}
H_{k}(\eta ; t)=0, \quad k \geq 2, \\
H_{1}(\eta ; t)=\sum_{i=1}^{N}\left(-n_{i} \log a_{i, N}(t)+\log \left(n_{i} !\right)\right), \quad \eta \in \Omega_{N}, t \geq 0 .
\end{gathered}
$$

In the context of stochastic models of coagulation and fragmentation, Gibbs distributions (2.1) with weights not depending on $t$ and $N$ emerged as early as the 1970s in the works of Kelly [16] and Whittle [24], devoted to reversible models of clustering at equilibrium. Vershik (see, e.g. [23]) who intensively studied such Gibbs distributions in the context of equilibrium models of an ideal gas, called them multiplicative measures. Pitman [21] introduced Gibbs processes of pure coagulation/fragmentatation on the state space $\Omega_{[N]}$, which is the set of all partitions of the set $[1, \ldots, N]$, while developing Kingman's theory of exchangeable partitons. Unlike in the present paper, Pitman's definition of the Gibbs process required that microcanonical (rather than canonical) distributions are Gibbsian at any time $t$. Such processes were subsequently extensively studied by Berestecky and Pitman [4], whose main result was the characterization of the weights of Gibbs fragmentation processes on $\Omega_{[N]}$. It turned out that the time reversal of these processes are CPs with $\psi(i, j)=a(i+j)+b$. (Note that, for $b \neq 0$, the latter rates are not of the form (2.1)). Goldschmidt et al. [10] constructed a Gibbs fragmentation process with weights that does not obey the characterization condition in [4]. This became possible because the constructed process does not possess the mean-field property. The interplay between the setup when the state space of a CP is set partitions and the setup in the present paper is discussed in more detail in [4], [12], and [21]. In [12], as a development of the idea in [14], a characterization of coagulation-fragmentation processes, such that the induced birth and death processes $\left|X_{N}^{(\rho)}(t)\right|$ are time homogeneous, was established. Based on this, a characterization of coagulation-fragmentation models, possessing time-independent microcanonical Gibbs distributions $\mathrm{P}\left(X_{N}^{(\rho)}(t)=\eta|| X_{N}^{(\rho)}(t) \mid=k\right)$, was obtained. By [11] and our theorem, the interrelation between CPs with Gibbsian canonical distributions and those with time-independent Gibssian microcanonical distributions is as follows. (We note that Gibbsian canonical distribution induces a Gibbsian microcanonical distribution, the latter 
being in general time dependent.) The CPs with $\psi(i, j)=a(i+j), a>0$, are the only ones that have Gibbsian canonical and time-independent Gibbsian microcanical distributions. On the other hand, the CPs with $\psi(i, j)=a(i+j)+b, a \geq 0, b>0$ are the only ones that have non-Gibbsian canonical distributions and time-independent Gibbsian microcanonical distributions, while the CPs with $\psi(i, j)=i f(j)+j f(i)$, when $f \neq$ constant are the only ones having Gibbsian canoninal and time-dependent Gibbsian microcanonical distributions. In conclusion, we mention a representation of Gibbs distributions (2.1) arising in the field of random combinatorial structures. In the case when the weights $a_{k, N}(t)$ do not depend on $t$ and $N$, the distributions (2.1) depict the distributions of the vectors $\eta=\left(n_{1}, \ldots, n_{N}\right) \in \Omega_{N}$ of component counts of random combinatorial structures (see, e.g. [2], [4], [8], [11], and [17]). In this setup $n_{k}$ stands for the number of nondecomposable components (e.g. cycles in a random permutation) of size $k$. A cornerstone fact in the theory of random structures is the representation of the aforementioned measures via the so-called conditional relation, which proved to be very useful for problems of asymptotic enumeration. The version of the conditional relation for time-dependent Gibbs distributions (2.1) reads as follows:

$$
p(\eta, \rho ; t)=\mathrm{P}\left(Z_{1, N}(t)=n_{1}, \ldots, Z_{N, N}(t)=n_{N} \mid \sum_{k=1}^{N} k Z_{k, N}(t)=N\right)
$$

for $\eta=\left(n_{1}, \ldots, n_{N}\right) \in \Omega_{N}$ and $t \geq 0$. Here $\left\{Z_{k, N}(t), k=1, \ldots, N, t \geq 0\right\}$ is the triangular array of Poisson random variables with parameters $a_{k, N}(t)$, such that the $Z_{k, N}(t), k=$ $1,2, \ldots, N$, are independent at any given time $t>0$. We note that in our setting

$$
\mathrm{P}\left(\sum_{k=1}^{N} k Z_{k, N}(t)=N\right)=(N !)^{-1} \exp \left(-\sum_{k=1}^{N} a_{k, N}(t)\right), \quad t>0 .
$$

\section{Acknowledgement}

We appreciate the constructive suggestions and critical remarks of the anonymous referee.

\section{References}

[1] Aldous, D. J. (1999). Deterministic and stochastic models for coalescence (aggregation, coagulation): a review of the mean-field theory for probabilists. Bernoulli 5, 3-48.

[2] Arratia, R., Barbour, A. D. and Tavaré, S. (2004). Logarithmic Combinatorial Structures: A Probabilistic Approach. European Mathematical Society, Zürich.

[3] Ball, J. M., Carr, J. And Penrose, O. (1986). The Becker-Döring cluster equations: basic properties and asymptotic behaviour of solutions. Commun. Math. Phys. 104, 657-692.

[4] Berestycki, N. And Pitman, J. (2007). Gibbs distributions for random partitions generated by a fragmentation process. J. Statist. Phys. 127, 381-418.

[5] Buffet, E. And Pulé, J. V. (1990). On Lushnikov's model of gelation. J. Statist. Phys. 58, 1041-1058.

[6] Buffet, E. And Pulé, J. V. (1991). Polymers and random graphs. J. Statist. Phys. 64, 87-110.

[7] Durrett, R., Granovsky, B. L. and Gueron, S. (1999). The equilibrium behavior of reversible coagulationfragmentation processes. J. Theoret. Prob. 12, 447-474.

[8] Freiman, G. and Granovsky, B. (2005). Clustering in coagulation-fragmentation processes, random combinatorial structures and additive number systems: asymptotic formulae and limiting laws. Trans. Amer. Math. Soc. 357, 2483-2507.

[9] Gnedin, A. And Pitman, J. (2006). Exchangeable Gibbs partitions and Stirling triangles. J. Math. Sci. 138, 5674-5685.

[10] Goldschmidt, C., Martin, J. B. and Spanó, D. (2008). Fragmenting random permutations. Electronic Commun. Prob. 13, 461-474.

[11] Granovsky, B. (2012). Asymptotics of counts of small components in random structures and models of coagulation-fragmentation. To appear in ESAIM Prob. Statist. 
[12] Granovsky, B. L. And Erlihson, M. M. (2009). On time dynamics of coagulation-fragmentation processes. J. Statist. Phys. 134, 567-588.

[13] Han, D., Zhang, X. S. And Zheng, W. A. (2008). Subcritical, critical and supercritical size distributions in random coagulation-fragmentation processes. Acta Math. Sinica (Engl. Ser.) 24, 121-138.

[14] Hendriks, E. M., Spouge, J. L., Elbi, M. And Schreckenberg, M. (1985). Exact solutions for random coagulation processes. Z. Phys. B 58, 219-227.

[15] Hu, C., Han, D. AND Hsu, C. (2007). Critical behavior of the heterogeneous random coagulation-fragmentation processes. J. Phys. A 40, 14649-14665.

[16] Kelly, F. P. (1979). Reversibility and Stochastic Networks. John Wiley, Chichester.

[17] Kolchin, V. F. (1999). Random Graphs (Encyclopedia Math. Appl. 53). Cambridge University Press.

[18] Lushnikov, A. A. (1977). Coagulation in finite systems. J. Colloid Interface Sci. 65, 276-285.

[19] Lushnikov, A. A. (1978). Certain new aspects of the coagulation theory. Atmos. Ocean. Phys. 14, 738-743.

[20] Marcus, A. H. (1968). Stochastic coalescence. Technometrics 10, 133-143.

[21] Pitman, J. (2006). Combinatorial Stochastic Processes (Lecture Notes Math. 1875). Springer, Berlin.

[22] Van Dongen, P. G. J. and Ernst, M. H. (1984). Kinetics of reversible polymerization. J. Statist. Phys. 37, 307-324.

[23] VershiK, A. M. (1996). Statistical mechanics of combinatorial partitions, and their limit configurations. Funct. Analysis Appl. 30, 90-105.

[24] Whittle, P. (1986). Systems in Stochastic Equilibrium. John Wiley, Chichester. 